\title{
Recombinant Fibroblast Growth Factor 4
}

National Cancer Institute

\section{Source}

National Cancer Institute. Recombinant Fibroblast Growth Factor 4. NCI Thesaurus. Code C1309.

A recombinant therapeutic agent which is chemically identical to or similar to endogenous fibroblast growth factor 4 (FGF-4). Therapeutic FGF-4 induces vascular permeability, angiogenesis, and arteriogenesis and has potential uses in the treatment of peripheral vascular disease. ( $\mathrm{NCIO4)}$ 